\title{
Optical Character Recognition based on Template Matching
}

\author{
By Md. Anwar Hossain \& Sadia Afrin \\ Pabna University of Science and Technology
}

Abstract- This paper presents an innovative design for Optical Character Recognition (OCR) from text images by using the Template Matching method.OCR is an important research area and one of the most successful applications of technology in the field of pattern recognition and artificial intelligence.OCR provides full alphanumeric visualization of printed and handwritten characters by scanning text images and converts it into a corresponding editable text document. The main objective of this system prototype is to develop a prototype for the OCR system and to implement The Template Matching algorithm for provoking the system prototype. In this paper, we took alphabet ( $A-Z$ and $a-z)$, and numbers (0-1), grayscale images, bitmap image format were used and recognized the alphabet and numbers by comparing between two images. Besides, we checked accuracy for different fonts of alphabet and numbers. Here we used Matlab R2018a software for the proper implementation of the system.

Keywords: OCR, template matching, grayscale images, binary images.

GJCST-C Classification: 1.2.7

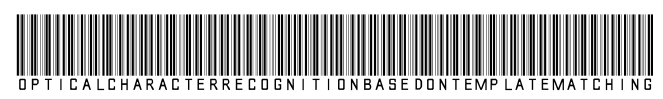

Strictly as per the compliance and regulations of:

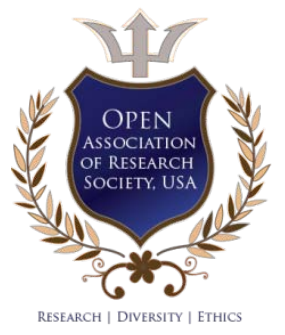

(c) 2019. Md. Anwar Hossain \& Sadia Afrin. This is a research/review paper, distributed under the terms of the Creative Commons Attribution-Noncommercial 3.0 Unported License http://creativecommons.org/licenses/by-nc/3.0/), permitting all non-commercial use, distribution, and reproduction inany medium, provided the original work is properly cited. 


\title{
Optical Character Recognition based on Template Matching
}

\author{
Md. Anwar Hossain ${ }^{\alpha} \&$ Sadia Afrin ${ }^{\sigma}$
}

Abstract- This paper presents an innovative design for Optical Character Recognition (OCR) from text images by using the Template Matching method.OCR is an important research area and one of the most successful applications of technology in the field of pattern recognition and artificial intelligence.OCR provides full alphanumeric visualization of printed and handwritten characters by scanning text images and converts it into a corresponding editable text document. The main objective of this system prototype is to develop a prototype for the OCR system and to implement The Template Matching algorithm for provoking the system prototype. In this paper, we took alphabet (A-Z and $a-Z)$, and numbers $(0-1)$, grayscale images, bitmap image format were used and recognized the alphabet and numbers by comparing between two images. Besides, we checked accuracy for different fonts of alphabet and numbers. Here we used Matlab R2018a software for the proper implementation of the system.

Keywords: OCR, template matching, grayscale images, binary images.

\section{INTRODUCTION}

$\mathrm{n}$ the era of modern technology, Optical character recognition has become one of the most significant topics. Optical Character Recognition is the Transformation of scanned images of printed, handwritten or typewritten text into machine-encoded text.

In the natural process, human eyes are an optical mechanism. The image which is seen by the eyes is the input for the brain.OCR functions like the human ability of reading. OCR is a system prototype which has its scope, and it is using the Template Matching algorithm that is applied to recognize the character.

In our paper, the key concept is OCR and Template Matching. Template Matching is the method which we use here for the proper implementation of OCR.

\section{a) Basic Concept of Optical Character Recognition (OCR)}

$\mathrm{OCR}$ is the technique which performs automatic identification and allows to automatically recognize the

\footnotetext{
Author a: Assistant Professor, Department of Information and Communication Engineering, Faculty of Engineering and Technology, Pabna University of Science and Technology, Pabna, Bangladesh. e-mail:manwar.ice@gmail.com

Author o: Student, Department of Information and Communication Engineering, Faculty of Engineering and Technology, Pabna University of Science and technology.e-mail: sadia.ice.pust@gmail.com
}

characters through an optical mechanism. The goal of OCR is to classify visual patterns corresponding to alphanumeric or another letter.OCR is wanted when the information should be readable both to a human and to a machine. Alternative input cannot be predefined.

\section{b) Basic Concept of Template Matching}

Template Matching is a technique for finding areas of an image that match a template. It is a high level machine visibility technique that destines the parts of an image that match a predefined template. It is flexible and relatively straight-forward to use which makes them one of the most well-known method.

It is the process of finding the location of a subimage called template inside an image. Once several the corresponding templates is found, then the centers are used as similar points to determine the registration parameter. Template Matching involves determining the similarities between a given pattern and windows of the same size in an image and identifying the window that produces the highest similarities measure.

It works by comparing derived image features of the image and the template for each possible displacement.

\section{Previous Work}

There are several approaches for text recognition and detection in images and videos have proposed earlier. These approaches can classify into two main classes: connected component based methods and texture based methods. A several numbers of research work on mobile OCR have founded. Motorola China Research center has presented a camera-based mobile OCR system for camera phones in[1].

An automatic text extraction system raised in [2]. An outline for a prototype Kanji OCR for recognizing machine printed Japanese texts and translated them into English is proposed in [3]. An approach of a character recognition system for Chinese script is in[4].

A system developed for only English capital letters in[5].A first skew correction technique for Camera Captured Business Card Images for Mobile Devices is proposed in[6].Optical Character Recognition remains very difficult for many languages. 


\section{IMPLEMENTATION}

OCR takes input which is a text image and gives editable text document as output. The classification process of OCR is mainly two types. They are i) training and ii) testing.

There are four steps in the OCR system. They are Pre-processing, Feature Extraction, Feature Training, and Feature Matching.

\section{a) Pre-processing}

Here, the text image is a grayscale image which is converted into a binary image. A Binary image is worked with the pixel value 0 , and 1 . In the binary images, the letters constitute by binary 1 (one) and the background constitute by binary 0 (zero).

b) Feature Extraction

The feature Extraction technique applies for all individual extracted character from the text image in the preprocessing steps. The objects which contain the pixel value fewer than 30 pixels will be removed.

A flow diagram of the OCR system is given below:

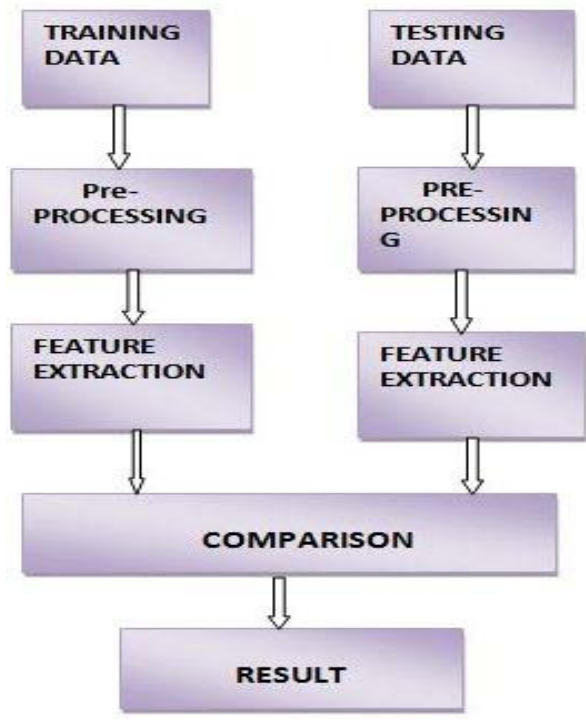

Fig. 1: Flow diagram of the OCR system

c) Feature Training

Here mainly two fonts namely Calibri (body) and Verdana have been considered for training data set. We will test the accuracy for other different fonts.

\section{d) Feature Matching}

It is the comparison stage. When the feature value is matched with the trained feature, then the matched feature is set to recognize the exact character. The unmatched characters are considered as the defective characters.

\section{e) Algorithm for Template Matching Method}

The main algorithm of this system prototype is the Template Matching algorithm. It is given below:
STEP 1: The character image from the detected string is selected.

STEP 2: The image to the size of the first template is rescaled.

STEP 3: After rescaling the image to the size of the first template (original) image, the matching metric is computed.

STEP 4: The highest match found is stored.

STEP 5: If the image is not matched, repeat step 3

STEP 6: The index of the best match is stored as the recognized character.

\section{f) Flow Chart for Template Matching}

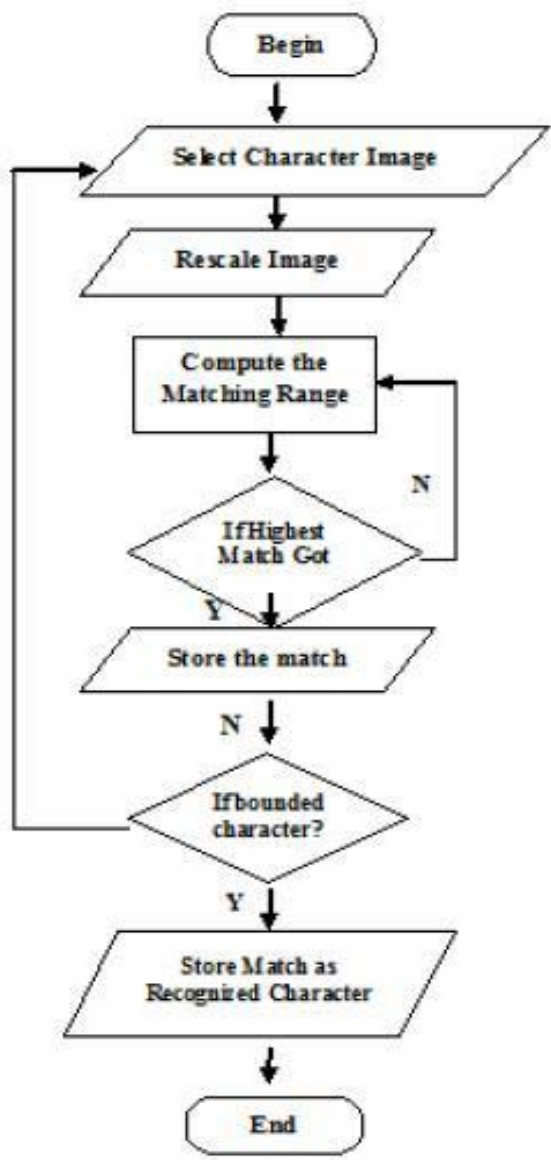

Fig. 2: Flow Chart for Template Matching

\section{Experimental Result}

The experiment performed by giving any still images with fixed font size as input, and the following output is found. In the following experimental result, we can see that all the letters are visible. The Font named as Calibri and Verdana is considered as training data here. 
a) Input Image

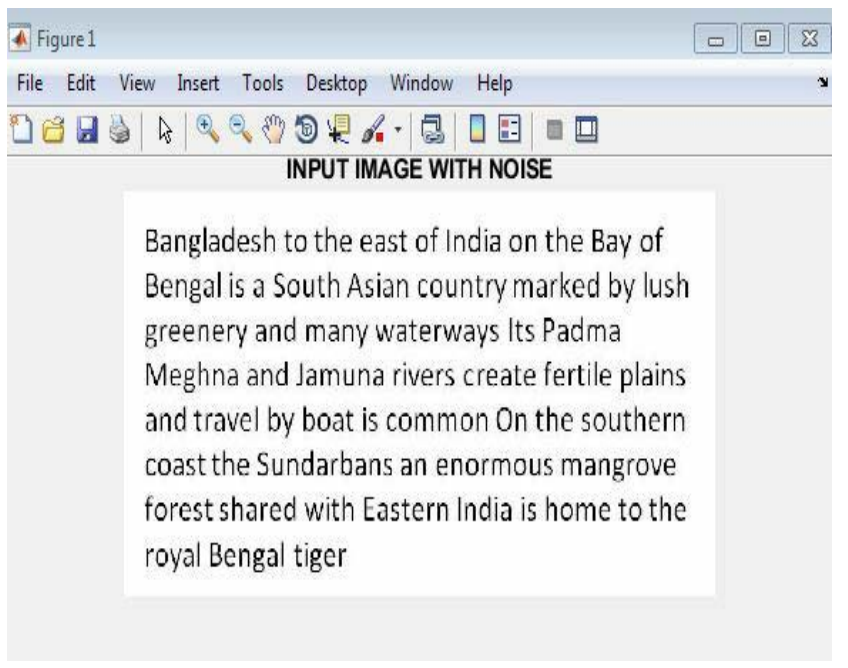

Fig. 3: Input Image

\section{b) Output Text Document}

\section{text.txt - Notepad}

File Edit Format View Help

Bangladesh to the east of India on the Bay of

Bengal is a South Asian country marked by lush

greenery and many waterways Its Padma

Meghna and Jamuna rivers create fertile plains

and travel by boat is common on the southern

coast the sundarbans an enormous mangrove

forest shared with Eastern India is home to the

royal Bengaj tiger

\section{Fig. 4: Output Text Document}

Based on Training data set, five cases each comprising of different text images (different alphabet and numbers) with seven different font is tested for OCR. Correctly and incorrectly clarified letters are counted, and the accuracy for each font is calculated as shown in the following Table:

Table 1: Table for OCR

\begin{tabular}{|c|c|c|c|c|}
\hline Test Image & Font Name & $\begin{array}{c}\text { Correct } \\
\text { Recognition }\end{array}$ & $\begin{array}{c}\text { Incorrect } \\
\text { Recognition }\end{array}$ & Accuracy \\
\hline \multirow{7}{*}{$\begin{array}{c}\text { Case- } 1 \\
\text { (Consist of } 10 \text { characters) }\end{array}$} & Calibri & 10 & 0 & $100 \%$ \\
\hline & Verdana & 9 & 1 & $90 \%$ \\
\hline & Arial & 7 & 3 & $70 \%$ \\
\hline & Berlin Sans & 6 & 4 & $60 \%$ \\
\hline & Cambria & 4 & 6 & $40 \%$ \\
\hline & Lucida Fax & 7 & 3 & $70 \%$ \\
\hline & Times new Roman & 6 & 4 & $60 \%$ \\
\hline \multirow{7}{*}{$\begin{array}{c}\text { Case-2 } \\
\text { (Consist of } 20 \text { characters) }\end{array}$} & Calibri & 20 & 0 & $100 \%$ \\
\hline & Verdana & 16 & 4 & $80 \%$ \\
\hline & Arial & 17 & 3 & $85 \%$ \\
\hline & Berlin Sans & 14 & 6 & $70 \%$ \\
\hline & Cambria & 13 & 7 & $65 \%$ \\
\hline & Lucida Fax & 15 & 5 & $75 \%$ \\
\hline & Times new Roman & 7 & 13 & $35 \%$ \\
\hline \multirow{7}{*}{$\begin{array}{c}\text { Case-3 } \\
\text { (Consist of } 25 \quad \text { characters) }\end{array}$} & Calibri & 24 & 1 & $96 \%$ \\
\hline & Verdana & 20 & 5 & $80 \%$ \\
\hline & Arial & 18 & 7 & $72 \%$ \\
\hline & Berlin Sans & 15 & 10 & $60 \%$ \\
\hline & Cambria & 14 & 11 & $56 \%$ \\
\hline & Lucida Fax & 18 & 7 & $72 \%$ \\
\hline & Times new Roman & 16 & 9 & $64 \%$ \\
\hline \multirow{7}{*}{$\begin{array}{c}\text { Case- } 4 \\
\text { (Consist of } 30 \text { characters) }\end{array}$} & Calibri & 30 & 0 & $100 \%$ \\
\hline & Verdana & 25 & 5 & $83.3 \%$ \\
\hline & Arial & 26 & 4 & $86.66 \%$ \\
\hline & Berlin Sans & 19 & 11 & $63.33 \%$ \\
\hline & Cambria & 15 & 15 & $50 \%$ \\
\hline & Lucida Fax & 24 & 6 & $80 \%$ \\
\hline & Times new Roman & 17 & 13 & $56.66 \%$ \\
\hline \multirow{7}{*}{$\begin{array}{c}\text { Case- } 5 \\
\text { (Consist of } 62 \text { characters) }\end{array}$} & Calibri & 61 & 1 & $98.38 \%$ \\
\hline & Verdana & 62 & 0 & $100 \%$ \\
\hline & Arial & 48 & 14 & $77.41 \%$ \\
\hline & Berlin Sans & 46 & 16 & $74.19 \%$ \\
\hline & Cambria & 35 & 27 & $56.45 \%$ \\
\hline & Lucida Fax & 40 & 22 & $64.51 \%$ \\
\hline & Times new Roman & 32 & 30 & $51.61 \%$ \\
\hline
\end{tabular}




\section{c) Graphical representation for All Cases}

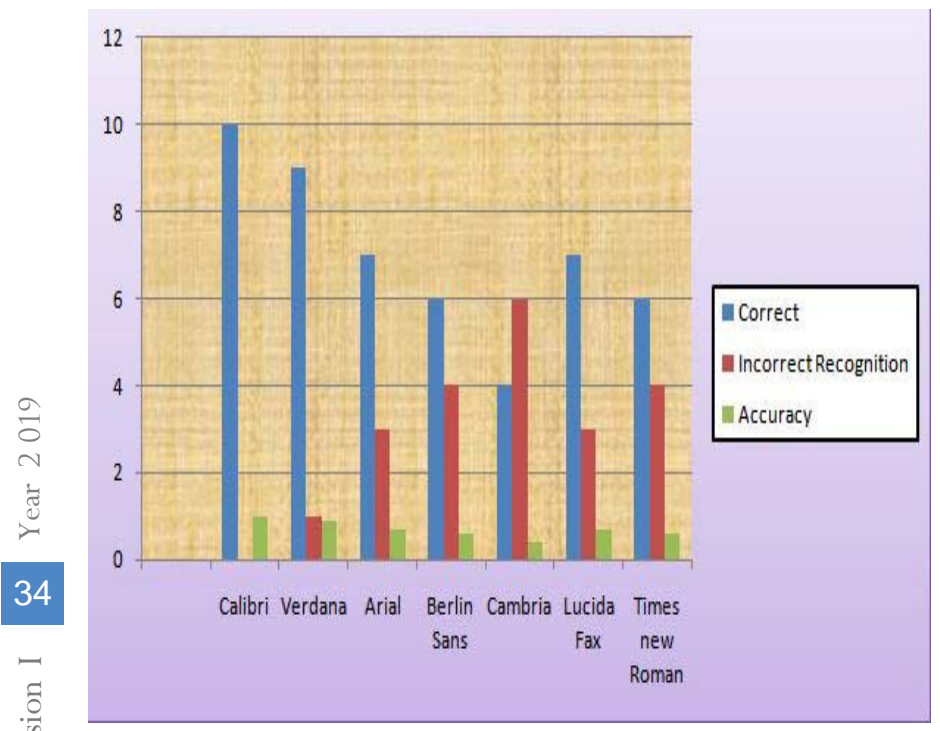

Fig. 5: Graph for Case-1

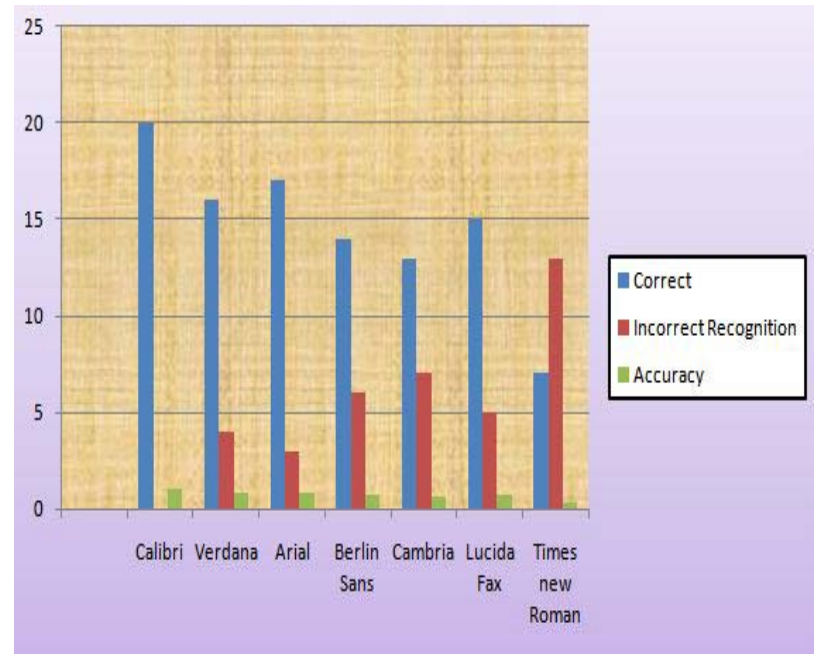

Fig. 6: Graph for Case-2

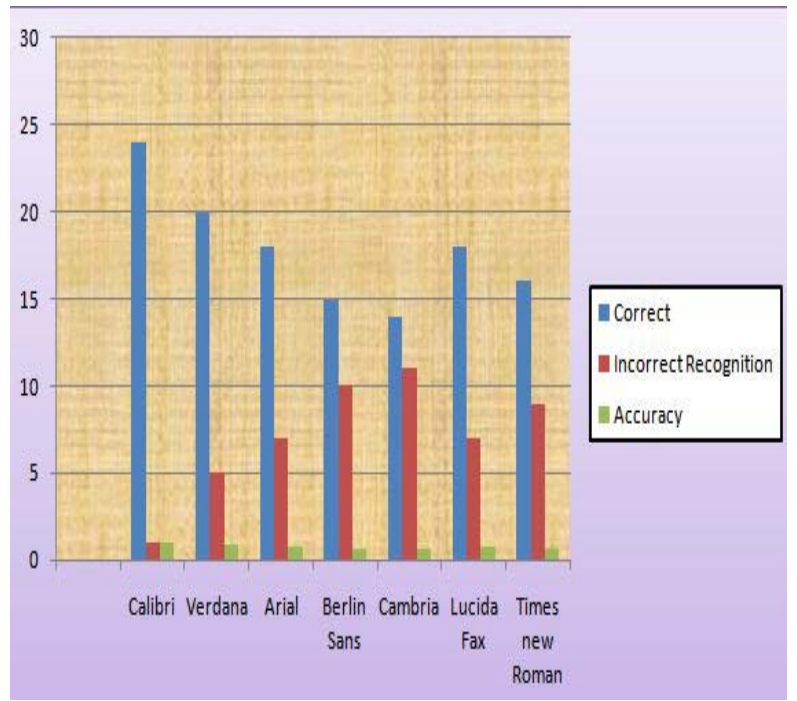

Fig. 7: Graph for Case-3

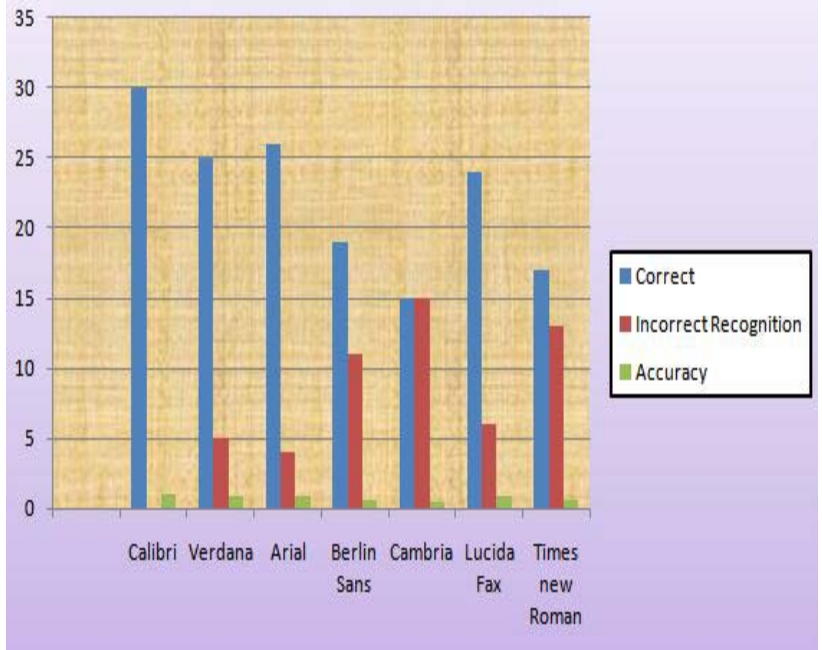

Fig. 8: Graph for Case-4

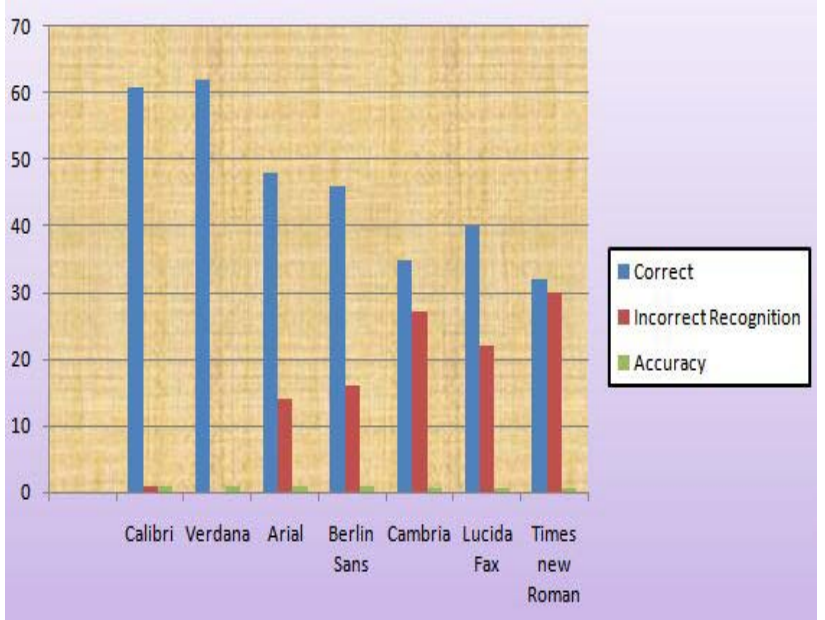

Fig. 9: Graph for Case-5

V. Discussion based on Experimental

\section{Result}

From above all the experimental result, we can reach a point of discussion. Although we have taken only two fixed sizes of fonts that is Calibri and Verdana as training data set, we have tested the accuracy for others five fonts namely Arial, Berlin Sans, Cambria, Lucida Fax and times new Roman. Experimental result on a set of images shows accuracy up-to $100 \%$ for Calibri, $100 \%$ for Verdana, $86.66 \%$ for Arial, $80 \%$ for Lucida Fax. Accuracy for Cambria and Times New Roman is very poor. We can improve the accuracy of these fonts by training the system with the character set of this fonts.

\section{Vi. Conclusions and Future Work}

There are several methods that have been introduced by different authors for optical character recognition. We serve a new technique to extract features from the images and identification of exact 
character to produce text document. As an overall view of the system prototype, we can say that we develop this system prototype by using the technique that is the Template Matching approach to identify the character image. The interface of the system prototype looks very simple and makes the user of this system prototype easier to use it. For this reason, the recognition process of this system becomes user-friendly and smoothly because of the steps used in this system while recognizing the characters.

In future work of this proposed algorithm can be enlarged and it will help the community in the field of handwritten character recognition. Except of alphabet and numbers, we can exalt the system with other characters. By ushering more features, we can enhance the accuracy.

\section{References Références Referencias}

1. X. Luo, J. Li and L. Zhen, "Design and implementation of a card reader based on build-in camera", International Conference on Pattern Recognition, pp. 417-420, 2004.

2. V. Wu, R. Manmatha and E. M. Riseman, "Finding Textin Images", In Proc. of Second ACM International Conference on Digital Libraries, Philadelphia, PA, pp.23-26, 1997. [2] K. S. Bae, K. K. Kim, Y. G. Chung and W. P. Yu,

3. M.Koga, R.Mine, T.Kameyama, T.Takahashi, M.Yamazakiand, T.Yamaguchi," Camera Based Kanji OCR for Mobile-phones: Practical Issues", Proceedings of the Eighth International Conference on Document Analysis and Recognition, pp.635639, 2005.

4. K. S. Bae, K.K.Kim, Y.G.Chung and W.p.Yu, "Character Recognition System for Cellular Phone with Camera", Proceeding of the 29th Annual International Computer Software and Applications Conference, vol.1, pp.539-544, 2005.

5. M. Laine and O.S.Nevalainen," A standalone OCR system for mobile camera-phones", Personal, Indoor and Mobile Radio Communications, 2006 IEEE 17th International Symposium, pp.1-5, Sept.2006.

6. A.F. Mollah, S.Basu, N.Das, R.Sarker, M.Nasipuri, M.Kundu," A Fast Skew Correction Technique for Camera Captured Business Card Images", Proc. Of IEEE INDICON-2009, pp.629-632, 18-20 December, Gandhinagar, Gujrat.

7. Jesse Hansen, "A Matlab Project in Optical Character Recognition (OCR)".

8. Thomas Natschlager "Optical Character Recognition", Institute of Theoritical Computer Science.

9. AparnaVara LakshmiVemuri, T.V.Saikrishna, AtulNegi, "Dataset Generation for OCR"
10. Nadira Muda, NikKamariahNik Ismail, SitiAzami Abu Bakar, Jasni Mohamad Zain "Optical Character Recognition By Using Template Matching (Alphabet)".

11. Line Eikvil (1993), "Optical Character Recognition".

12. Quin Chen (2003), "Evaluation of OCR Algorithms for Images with Different Spatial Resolutions and Noises", University of Ottawa.

13. Rachit Virendra Adhvaryu "Optical Character Recognition Using Template Matching". 